\title{
New Bioactive Molecules with Potential Antioxidant Activity from Various Extracts of Wild Edible Gelam Mushroom (Boletus spp.)
}

\author{
M. H. M. Y. Yuswan'1, Jameel R. Al-Obaidi², A. Rahayu'1, S. Sahidan¹, F. Shazrul', D. Fauzi' \\ ${ }^{1}$ School of Bioscience and Biotechnology, National University of Malaysia, Selangor, Malaysia \\ ${ }^{2}$ Agro-Biotechnology Institute, c/o MARDI Headquarters, Selangor, Malaysia \\ Email: fauzid@ukm.my
}

Received 2 March 2015; accepted 20 April 2015; published 24 April 2015

Copyright (C) 2015 by authors and Scientific Research Publishing Inc.

This work is licensed under the Creative Commons Attribution International License (CC BY). http://creativecommons.org/licenses/by/4.0/

(c) $\underset{\mathrm{EY}}{\mathrm{B}}$ Open Access

\section{Abstract}

Boletus spp. is a wild edible Gelam mushroom, which is only available at coastal area of Bachok, Kelantan. Locals have used this mushroom as a food source and as a traditional medicine. In this study, antioxidants were extracted using Mili-Q water and methanol. Extraction of $1.0 \mathrm{~g}$ of Boletus spp. mushroom pileus and stipe by water produced $0.22 \pm 0.09 \mathrm{~g}$ and $0.3 \pm 0.07 \mathrm{~g}$ of total aqueous soluble extracts, respectively. Methanol extraction of $1.0 \mathrm{~g}$ Boletus spp. mushroom pileus and stipe produced $0.31 \pm 0.08 \mathrm{mg}$ and $0.37 \pm 0.05 \mathrm{mg}$ of total methanol soluble extracts, respectively. DPPH assay has been used to determine the value of antioxidant efficiency of each extracts. DPPH assay revealed that the $\mathrm{EC}_{50}$ value of pileus aqueous soluble extract and both pileus and stipe methanol soluble extracts were $1.2 \mathrm{mg} / \mathrm{ml}$. The $\mathrm{EC}_{50}$ value of stipe aqueous soluble extracts was $1.4 \mathrm{mg} / \mathrm{ml}$. The $\mathrm{EC}_{50}$ value of ascorbic acid, an antioxidant control was $0.5 \mathrm{mg} / \mathrm{ml}$. Liquid chromatographyDPPH (LC-DPPH) assay combined with Quadrupole Time-of-flight Mass Spectrometry (QTOF-MS) analysis of 3:2 ratio methanol and water extract has detected 43 antioxidant compounds that were involved in the reduction of DPPH. To identify these compounds, SIMCA 3.0 software that consists of PCA, OPLS-DA and s-plot analyses had been used. PCA analysis had shown the antioxidant activity from sample on DPPH as $\mathbf{7 3 . 6 \%}$. This antioxidant activity confirmed by OPLS-DA analysis had shown the variability between sample and control as $99.6 \%$ with $99.3 \%$ prediction. S-plot selection had shown ten antioxidant compounds significantly involved in the DPPH assay. Four of these compounds were identified by the METLIN and NIST databases. The antioxidant metabolites were 2,4,6-trimethylacetophenone imine, glutamyl tryptophan, azatadine and lithocholic acid glycine conjugate. In conclusion, this study revealed that Boletus spp. mushroom is rich with natural antioxidants, which are potentially useful for multiple nutritional and health applications. 


\section{Keywords}

\section{Boletus, DPPH, EC ${ }_{50}$, Chemometric, QTOF, PCA, OPLS-DA and S-Plot}

\section{Introduction}

Number of chronic human diseases such as cardiovascular and cancer are increasing globally. Besides genetic inheritance factor, several publications designate that one of the most important factor of causing human diseases is posed by uncontrolled production of free radicals, very active by-products having one or more unpaired electrons [1]-[5]. As a result, these free radicals will accumulate in the body and leading to those diseases when the free radicals abundance in the human body becomes unbalance [6].

Alongside the harm that free radicals can persuade in biological systems, they are major culprits of food corrosion, loss of colour, taste, and diet value. Artificial antioxidants, used in the processed food as food-preserving, are being limited due to their hazard of carcinogenicity [7]. For these reasons, many consumers prefer natural food additives, such as extracts from fruits and vegetables, over synthetic ones. Isolation of natural antioxidants from plants or fungi for food applications is therefore necessary.

A big number of fungal-derived bioactive compounds, both cellular components and secondary metabolites, have been shown to affect the human immune system and could be used to treat a variety of diseases [1]. Recent increase in the application of bioactive compounds produced by white rot fungi in the food processing or pharmaceutical industry [8] is activating a global search for new natural bioactive compounds of fungal origin. Moreover, their production has become an important field of contemporary biotechnology.

Consequently, extensive studies have been conducted to find and test the antioxidant compounds especially from natural resources for instance mushrooms. Mushrooms have been reported as therapeutic food in order to prevent several diseases such as hypertension, hypercholesterolemia, atherosclerosis and cancer [8]-[11]. These therapeutic functions are mainly due to mushrooms' chemical composition [12].

Boletus spp. are basidiomycete fungi comprising over 100 species distributed around the world [13]. Some of them are edible and have valuable health value such as low in calories and fat besides high in carbohydrate, protein, mineral and vitamin [12]-[14]. One of them is known as Gelam mushroom (Boletus spp.) which is a wild edible mushroom from Kelantan state in the north-east of Peninsular Malaysia. Local people claim that this mushroom can treat cancer disease and lowering blood sugar level. However, there is no scientific study conducted yet to support these claim.

Mushrooms have powerful antioxidant properties derived from different compounds such as selenium, ergothioneine, and phenolics. Mushrooms are considered to be a good source of phenolic antioxidants, such as variegatic acid and diboviquinone [15]. Some reports dealing with these phenolic compounds report on less antioxidant activity of diboviquinone in comparison to variegatic acid [16]. Nowadays, mushrooms are known as a useful food and source of physiologically valuable components that could be of great interest.

Considering the growing interest for mushrooms, as dietary source for human consumption, and the demand search of natural antioxidants sources, the aim of this study was to determine the $\mathrm{EC}_{50}$ value of various extract from Boletus spp. for their potential antioxidant activity and in order to analyze the potential antioxidant activity of different extracts from the Boletus spp. using LC-QTOF-MS.

\section{Materials and Methods}

\subsection{Chemicals}

Methanol and acetonitrile (both HPLC grade) was purchased from Aldrich-Sigma and Mili-Q water was produced by Mili-Q Integral 5 water purification system. For mobile phase additive, formic acid (purity $\geq 99 \%$ ) was used and purchased from Aldrich-Sigma.

\subsection{Sample Collection and Treatment}

Boletus spp. was collected at Bachok $\left(6.0667^{\circ} \mathrm{N}, 102.4000^{\circ} \mathrm{E}\right)$, Kelantan. All the samples were cleaned by brushing the entire sample surface before quenching into liquid nitrogen to bring back to lab. In the lab, all the 
samples were separated to pileus and stipe prior to lyophilization using freeze dryer (Freezone 4.5, Labconco, Kansas City, MO, USA; temperature: $-40^{\circ} \mathrm{C}$ ). Afterwards, all the samples were pulverized using UDY cyclone mill to make fine powder sample and stored at $-80^{\circ} \mathrm{C}$ until use.

\subsection{Sample Extraction for DPPH Oxidation Assay}

Sample preparation was carried out as described by Wong and Chye [17] with some modification. $1 \mathrm{~g}$ of stipe fine powder sample was weighed and transferred into $250 \mathrm{~mL}$ Erlenmeyer flask containing $5 \mathrm{~mL}$ of methanol. The mixture was incubated by shaking at ambient temperature in dark environment for overnight. Subsequently, the mixture was filtered by using what man No.1 filter paper to separate the extract from the sample residue. The extract was concentrated by rotary evaporator (IKA ${ }^{\circledR}$ HB10, CB-1906, Janke \& Kunkel-IkaLabortechnik, Germany) under reduced pressure at $60^{\circ} \mathrm{C}$. The crude extract was stored in $-20^{\circ} \mathrm{C}$ for next uses. All the extraction steps were repeated but methanol was substituted by water and was boiled for 10 minutes prior to incubation. Freeze dried technique was applied instead of rotary evaporator to concentrate the water extract. Each extract was performed in five replicates and yield of extraction was reported as mean \pm standard deviation value. All the extraction technique was repeated using pileus fine powder sample instead of stipe fine powder sample.

\subsection{DPPH Oxidation Assay}

DPPH oxidation assay was performed according to Calderón et al. [18], Wong \& Chye [17] and Mujic et al. [19] with some modifications. $1 \mathrm{ml}$ of methanol or water extract $(1 \mathrm{mg} / \mathrm{ml}$ to $5 \mathrm{mg} / \mathrm{ml})$ was mixed with $1 \mathrm{ml}$ of 0.1 $\mathrm{mM}$ DPPH in $15 \mathrm{ml}$ falcon tube. The mixture was shaken vigorously and incubated at ambient temperature for 15 minutes. Next, the absorbance of mixture was determined by using Cary ${ }^{\circledR}$ Bio UV-visible spectrophotometer (Varian, Australia) at $515 \mathrm{~nm}$. In order to zero the absorbance, methanol was used as blank. Mixture without any type of extract and ascorbic acid were used as control and standard, respectively. The antioxidant activity of methanol or water extract was calculated as follow:

$$
\text { Antioxidant activity }(\%)=[(\mathrm{A} 0-\mathrm{A} 1) / \mathrm{A} 0] \times 100 \%
$$

where as $\mathrm{A} 0$ is the absorbance of the control and $\mathrm{A} 1$ is absorbance of the sample.

\subsection{DPPH Oxidation Assay for LC-QTOF-MS}

The sample preparation for LC-TOF-MS screening of DPPH oxidation assay was conducted according to Calderón et al. [18] and Gummer et al. [20] with some modification. $150 \mathrm{mg}$ of fine powder sample was weighed and transferred into pre-chilled $2 \mathrm{ml}$ microcentrifuge tube. $600 \mu \mathrm{l}$ of pre-chilled methanol was added and shaken vigorously by using vortex prior to incubate for overnight at $-20^{\circ} \mathrm{C}$. After that, the supernatant was separated from its residue by centrifuging at 10,000 rcf for 15 minutes. The supernatant was transferred into a new pre-chilled $2 \mathrm{ml}$ microcentrifuge tube and stored at $-20^{\circ} \mathrm{C}$ until for next use. The residue was re-dissolved by adding $400 \mu \mathrm{l}$ of pre-chilled Mili-Q water and shaken vigorously by using vortex. The mixture was incubated for overnight at $4^{\circ} \mathrm{C}$. Subsequently, the supernatant was separated from its residue by the same technique as methanol solvent. The supernatant was transferred into the previous $2 \mathrm{ml}$ microcentrifuge tube containing the methanol extraction and shaken gently before adding $1 \mathrm{ml}$ of $1 \mathrm{mg} / \mathrm{ml}$ of DPPH in methanol solvent. After 5 minutes, the mixture was filtered and immediately analyzed by injecting into the LC-QTOF-MS.

\subsection{LC-QTOF-MS Instrumentation for DPPH Oxidation Assay}

This study was performed according to Hajji et al. [21] and Vaclavik et al. [22] with some modification. The LC-QTOF-MS used in this study was an Agilent 1290 liquid chromatography consist of binary pump, autosampler, column thermostat compartment and diode array detector hyphenated to an Agilent 6550 mass spectrometry QTOF equipped with electrospray ionization (ESI) via Jet Stream Technology (Agilent Technologies, Santa Clara, CA, USA). The matrix separation was conducted via the reversed phase Zorbax Eclipse Plus C18, $2.1 \mathrm{~mm} \times 100 \mathrm{~mm}, 1.8 \mu \mathrm{m}$ particle size (Agilent Technologies, Santa Clara, CA, USA). Mili-Q water with 0.1\% of formic acid (A) and acetonitrile with $0.1 \%$ formic acid (B) were used as the mobile phase with linear gradient from $5 \%-95 \%$ of B for 19 minutes at flow rate $0.5 \mathrm{ml} / \mathrm{min}$. The injection volume was set to $1 \mu \mathrm{l} \mathrm{per} \mathrm{sample.}$ Then, all the samples were ionized at positive mode ionization and continuous internal calibration was per- 
formed during all chromatographic separations, comprising of protonated purine and protonated hexakis (2,2difluoroethoxy) phosphazine signals, m/z 121.0509 and m/z 922.0098, respectively. The drying gas temperature was set to $225^{\circ} \mathrm{C}$, nozzle voltage to $2000 \mathrm{~V}$, sheath gas temperature $275^{\circ} \mathrm{C}$, sheath gas flow 12 liter/min, drying gas flow to 13 liter/min, nebulizer pressure to $20 \mathrm{psi}$, capillary voltage to $4000 \mathrm{~V}$ and spectra acquisition rate to 4 spectra/second. As comparison, Mili-Q water was used as blank to detect any background or impurity peaks whereas sample without DPPH in methanol solvent was used as control. All the samples were prepared for eight biological replicates prior to injection with three technical replicates.

\subsection{Antioxidant Metabolites Identification}

Antioxidant metabolites were identified by comparing the mass spectral and retention time of detected compounds with those following database: Agilent METLIN database and National Institute of Standards and Technology (NIST).

\subsection{Data and Statistical Analysis}

Masshunter Workstation software (version 4.0 Qualitative Analysis, Agilent Technologies, Santa Clara, CA, USA) consists of Qualitative Analysis B.05.00 (MQA) and Mass Profiler Professional B.12.6 (MPP) was used for processing full single MS data. Data analysis was started by using MQA through specific algorithm (intensity threshold: 1000 cps, retention time: 1 - 23 minutes) to extract ions from the full single MS data in form of molecular features (MFs). Background subtraction was also performed in order to remove the impurity or contaminant peaks base on blank chromatogram. Then, all the MFs were converted to compound exchange format (.cef files) to be exported to MPP for antioxidant metabolites profiling. Subsequently, MPP was used to analyze the (.cef files) through compounds alignment, filtering, significance testing and fold change. Results for MPP were used back in MQA to re-extract ions from the full single MS data instead of using previous algorithm to avoid false positive and proceed to MPP as mention previously. Afterwards, results from MPP were exported to SIMCA 13 to perform multivariate analysis including principle component analysis (PCA), orthogonal partial least square discriminant analysis (OPLS-DA) and s-plot.

\section{Results and Discussions}

\subsection{Yield of Methanol and Water Extract from Boletus spp.}

In order to determine active compounds in Boletus spp., extraction yield from methanol and water solvent are calculated from five replicates. As shown in Table 1, yield of extract percentage from fresh Boletus spp. is significantly high in methanol extract either for pileus or stipe compares to all type of water extract. This result is in line with those data obtained in previous studies done on different wild tropical edible mushroom like Pleurotus porrigens, Hygrocybe conica, Xerula furfuracea, Schizophyllum commune and Polyporus tenuiculus [17], Lentinus edodes and Volvariella volvacea [23] suggesting that most of the active compounds in Boletus spp. are organic compounds which soluble readily in methanol (organic solvent) but not in aqueous medium (water). Meanwhile, there is significant difference observed between extraction parts of Boletus spp. as well with stipe shows significantly high for yield of extract either for methanol or water extracts. This is presumably that the polar active compounds came from this part.

\subsection{DPPH Scavenging Activity of Boletus spp. Extract}

The free radical of 2,2-diphenyl-1-picrylhydrazyl is a stable free radical used to evaluate the scavenging activity Table 1. Yield of extraction for methanol and water extract at different part of Boletus spp., each sample with five replicates.

\begin{tabular}{ccc}
\hline \multirow{2}{*}{ Boletus spp. part } & \multicolumn{2}{c}{ Yield of extract (\% of fresh Boletus spp.) } \\
\cline { 2 - 3 } & Methanol extract & Water extract \\
\hline Pileus & $0.31 \pm 0.02^{\mathrm{a}, 2}$ & $0.22 \pm 0.02^{\mathrm{b}, 2}$ \\
Stipe & $0.37 \pm 0.10^{\mathrm{a}, 1}$ & $0.30 \pm 0.06^{\mathrm{b}, 1}$ \\
\hline
\end{tabular}

Within the row, mean \pm standard deviation values with different alphabetic superscript are significantly different at $\alpha=0.05$ for extraction solvent. Within the column, mean \pm standard deviation values with different numeric superscript are significantly different at $\alpha=0.05$ for Boletus spp. part. 
of sample extract [24]. In this study, all the sample extracts show positive scavenging activity against extract concentration until certain limit. Ascorbic acid shows the highest DPPH scavenging activity suggesting that it is still the best natural antioxidant metabolite with $\mathrm{EC}_{50}$ value is approximately $0.5 \mathrm{mg} / \mathrm{ml}$ (Figure 1). By looking at the sample extracts, methanol extracts either from pileus or stipe show higher DPPH scavenging activity compare to all type of water extracts. This may be due to the abundance of active compounds in methanol extracts as discuss before. However, the whole sample extracts show approximately $1 \mathrm{mg} / \mathrm{ml}$ for their $\mathrm{EC}_{50}$ value except for water extract from stipe. This result is close to Heleno et al. [25] findings where the $\mathrm{EC}_{50}$ value for six different Boletus species is between $0.3-0.8 \mathrm{mg} / \mathrm{ml}$. On the other hand, there is no significant difference observed between all types of sample extracts.

\subsection{Chromatographic Separation}

The matrix separation of samples, control and blank are carried out through reversed phase chromatographic separation that produces 24 total ion chromatograms (TIC), respectively. It was predicted that the intensity of sample chromatogram lower than control chromatogram; indicates some of antioxidant metabolites have been oxidized by the DPPH. Figure 2 showed that the TIC for sample, control and blank chromatogram. In general,

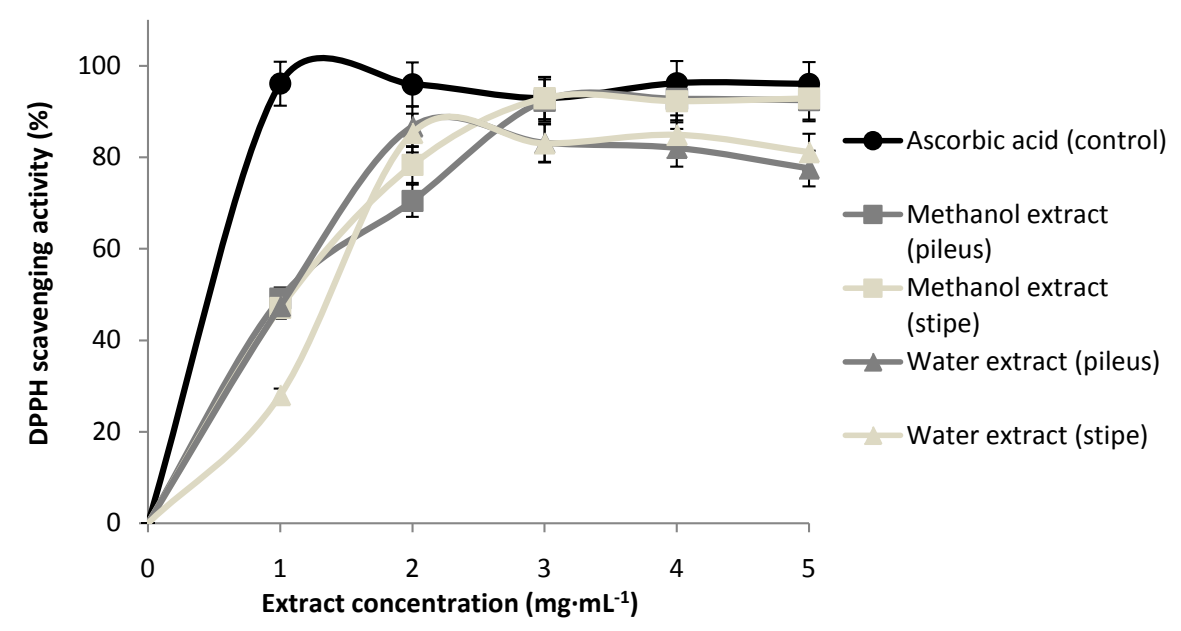

Figure 1. DPPH scavenging activity of water and methanol extract from Boletus spp. Each value is mean \pm standard deviation from five replicates.

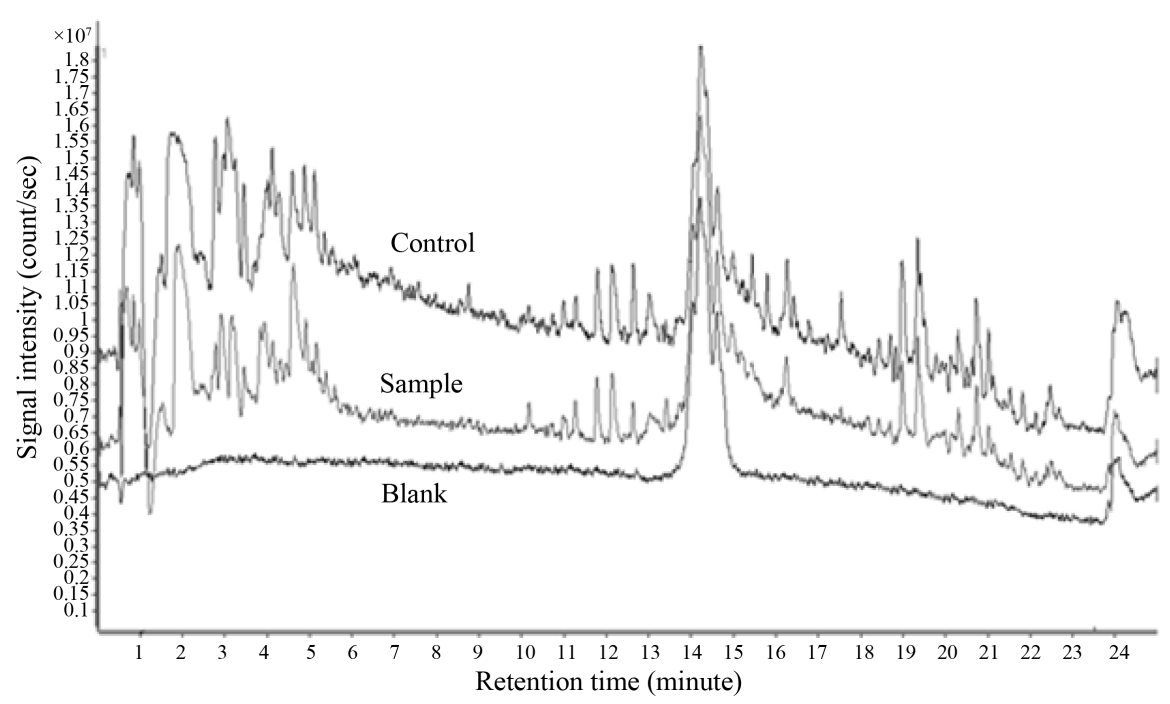

Figure 2. Total ion chromatogram (TIC) for sample, control and blank. The step gradient elution is between 1 - 21 minutes from $5 \%-95 \%$ of $\mathrm{B}$. 
the gradient of mobile phase was constantly decreased from polar to non-polar whereas the stationary phase was maintained to be non-polar, enable the hydrophilic antioxidant metabolites elute first followed by hydrophobic antioxidant metabolites. It is obvious that some peaks are disappeared and some are lower in the sample chromatogram when comparing to the control chromatogram at particular retention time, thus supporting the idea of oxidized antioxidant metabolites by the DPPH. This observation is similar to Calderón et al. [18]. However, there are few peaks observed from blank chromatogram as well. It could be due to some impurities from mobile or stationary phase even the LCMS system. That is a good practice to subtract the impurity peaks before extracting the ions from the full single MS data [18].

\subsection{Data Mining and Analysis}

In order to mining and analyzing the data obtained from chromatographic separation through LC-QTOF-MS, the full single MS data were exported to MQA prior to MPP to analyze the complexity of the data. Expectedly, number of MFs is drastically decreased across the sample set either for before or after validation stage (Table 2). The decrease number of MFs gives the idea of MFs accuracy after passing through the alignment, filtering, significance testing and fold change processes. The initial number of MFs (5376) is obtained after aligning all the suggested MFs from MQA across all sample and control. The number of MFs is reduced (969) after filtering by frequency; where the MFs are retained only if the previous MFs (5376) are 95\% existed in one group only. Subsequently, the number of MFs is continuing decreased (735) after statistically analyzed by un-paired t-test prior to volcano plot analysis (301) with fold change value is more than 7 fold. Interestingly, the real number of MFs (43) is one seventh from the number of MFs before validation (301); reveals that there are so much false positive. This may be due to the initial number of MFs suggested from MQA is extracted based on algorithm that is not correspond to the compounds present in sample and control.

\subsection{Advanced Chemometric Analysis}

To overview the relationship between samples and controls, PCA is employed to reveal the variation within and between variables in multidimensional space. PCA is an unsupervised recognition technique, enabling data dimensionality reduction while retaining maximum variability of the data [18]. As illustrated in Figure 3, score plot of PCA shows the samples and controls distribution. The PCA model contains three components where the first, second and third component explains $64.7 \%, 4.9 \%$ and $4.5 \%$ of the variation, respectively. Total of variation for the model is $74 \%$ and the other $26 \%$ is residue. As observed from Figure 3 , the samples and controls are separated between each other; indicate variation of antioxidant metabolites abundance. For the samples, there is within variation and two outliers as there are two dots located outside the eclipse Hoteling's $\mathrm{T}^{2}$. The within variation of samples may be due to antioxidant metabolic flux while reacting with DPPH, meanwhile the outliers may be due to human error while conduction the assay prior to LC-QTOF-MS analysis. On the other hand, the controls are pure sample without DPPH that explaining the cluster character of control.

In order to overview the variation of antioxidant metabolites abundance between samples and controls, loading plot of PCA is illustrated in Figure 4. There are two clusters of antioxidant metabolites observed which is from samples and controls where all the dots represent antioxidant metabolites. As noticed, the abundance of antioxidant metabolites from samples is less than controls. This may be due to some of the antioxidant metabolites of samples have been utilized to oxidize the DPPH.

To construct and validate a statistical model for antioxidant metabolites abundance classification, OPLS-DA is used to demonstrate the discrimination potential offered by PCA model. This statistical model is better than

Table 2. Advanced chemometric analysis of MFs by MPP.

\begin{tabular}{ccccc}
\hline \multirow{2}{*}{$\begin{array}{c}\text { Stage of MPP } \\
\text { process }\end{array}$} & Initial & Filter by frequency $^{\mathrm{b}}$ & One-way ANOVA $^{\mathrm{c}}$ & Filter by fold changed $^{\mathrm{d}}$ \\
\cline { 2 - 5 } & $5376^{\mathrm{a}}$ & 969 & 735 & 301 \\
Before validation & 242 & 151 & 133 & 43 \\
After validation & 242 & & Yield of extract (\% of fresh Boletus spp.) \\
\hline
\end{tabular}

${ }^{a}$ After impurities or contaminants subtraction from blank. ${ }^{b}$ Retain entities where at least $95 \%$ of sample in one group. ${ }^{c} \mathrm{p}$-value for before validation and after recursion stage of MPP process are $<0.001$ and $<0.005$, respectively. ${ }^{\mathrm{d}}$ Fold change for before and after validation stage of MPP process are $>7$, respectively. 


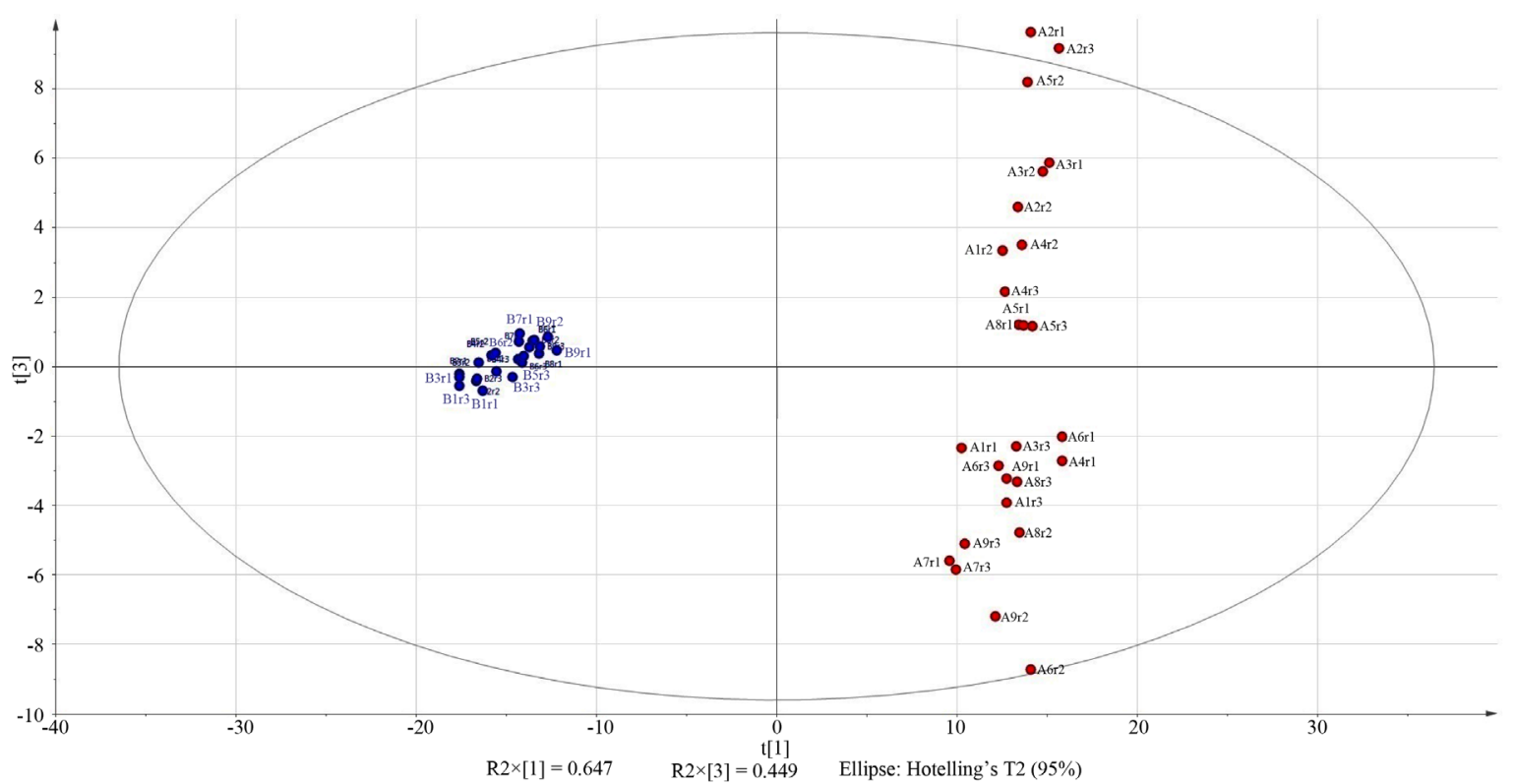

Figure 3. Score plot of PCA between samples and controls. Each sample and control with nine biological replicates and three technical replicates $(n=27)$.

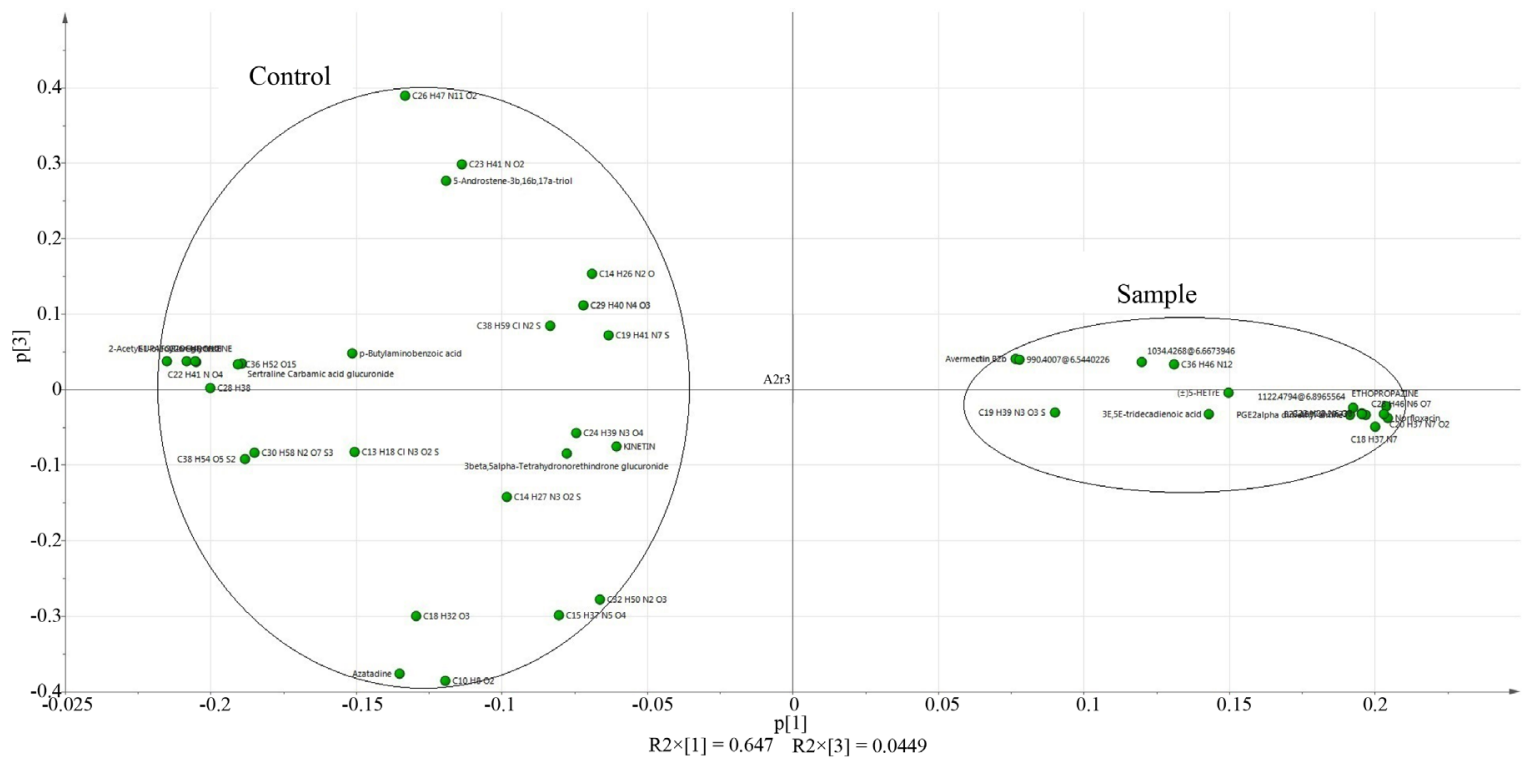

Figure 4. Loading plot of PCA for samples and controls. All the dots represent antioxidant metabolites either from sample or control.

PLS-DA which had been demonstrated by Vaclavik et al. [22] where the OPLS-DA improves model visualization and interpretation by separated the data into predictive (correlated) and orthogonal (uncorrelated) variation [26]. Score plot of OPLS-DA shows good separation with 99.6\% of variation between samples and controls with goodness of prediction is $99.3 \%$ (Figure 5). The OPLS-DA model contains one predictive variation and one orthogonal variation with $64.1 \%$ and $5 \%$, respectively. It means $64.1 \%$ of variation regarding to separation between samples and control, meanwhile the $5 \%$ of variation regarding to the antioxidant metabolites abundance.

In order to identify the antioxidant metabolites that involved in DPPH oxidation assay, s-plot is an easy way to visualize the OPLS-DA classification model [26]. The s-plot combined the modeled covariance and modeled correlation from OPLS-DA model in a scatter plot where if the data have variation in peak intensities, this plot will look like an S. As can be seen from Figure 6, all the dots represent antioxidant metabolites from samples 


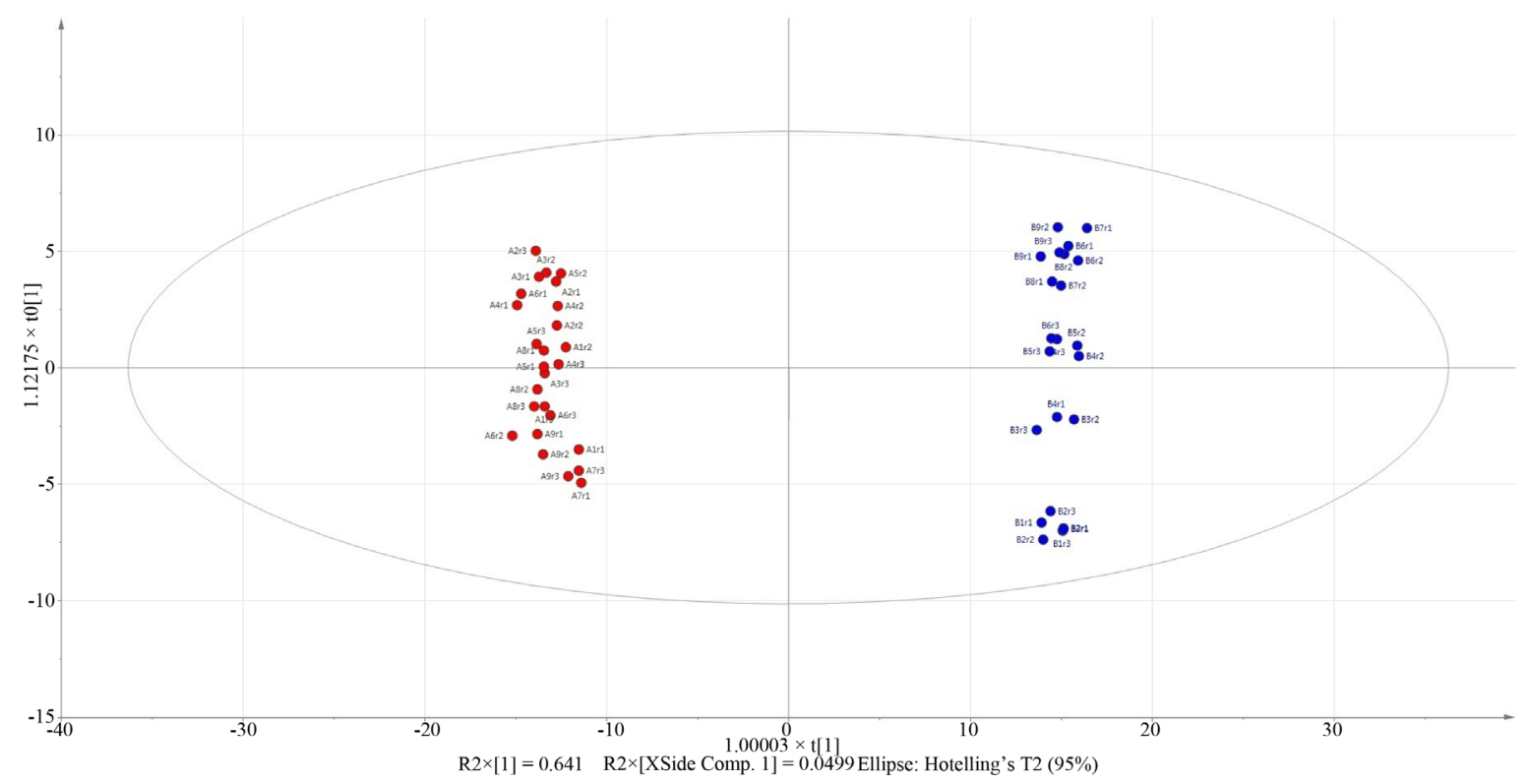

Figure 5. Score plot of OPLS-DA for samples and controls. Each sample and control with nine biological replicates and three technical replicates $(n=27)$.

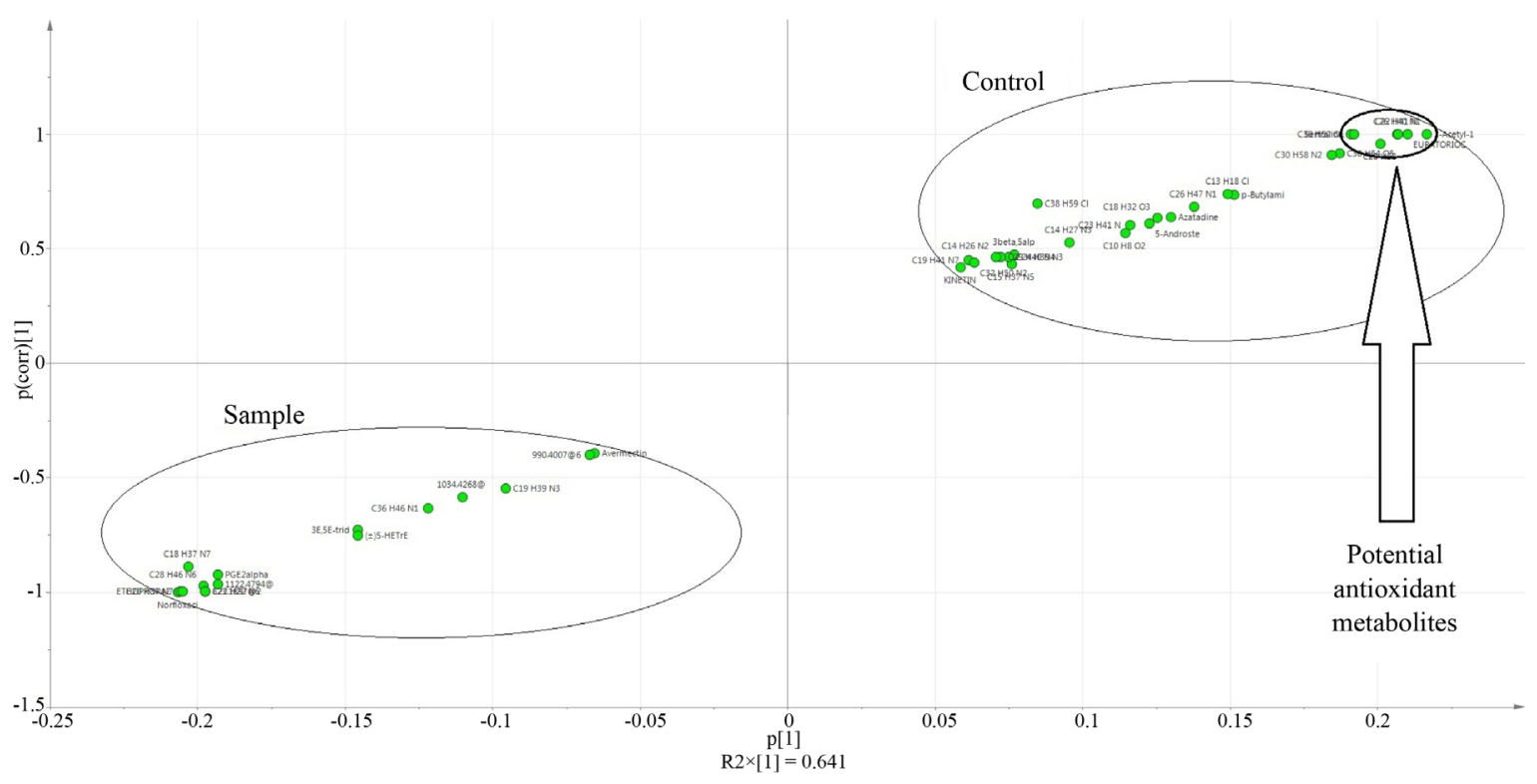

Figure 6. S-plot between sample and control. All the dots represent antioxidant metabolites either from sample or control.

and controls which look like the S shape. The $\mathrm{x}$-axis will describe the magnitude of each variable whereas the $\mathrm{y}$-axis represents the reliability of each variable. In this case, the potential antioxidant metabolites that involved in DPPH oxidation assay should come from control group at high magnitude and reliability (Table 3).

\section{Conclusion}

In this study, Boletus spp. extracts had showed antioxidant activity by the DPPH assay. To the best of our knowledge this study considered the first study that revealed the antioxidant activity of Boletus spp. proven by profiling the antioxidant metabolites through high-end LC-QTOF-MS. From the advanced chemometric analysis, all the potential antioxidant metabolites that involved in the DPPH oxidation assay were statistically significance. Therefore, Boletus spp. extract might be a potential source of natural antioxidants, and the consumption of mu- 
Table 3. Potential antioxidant metabolites involved in DPPH oxidation assay.

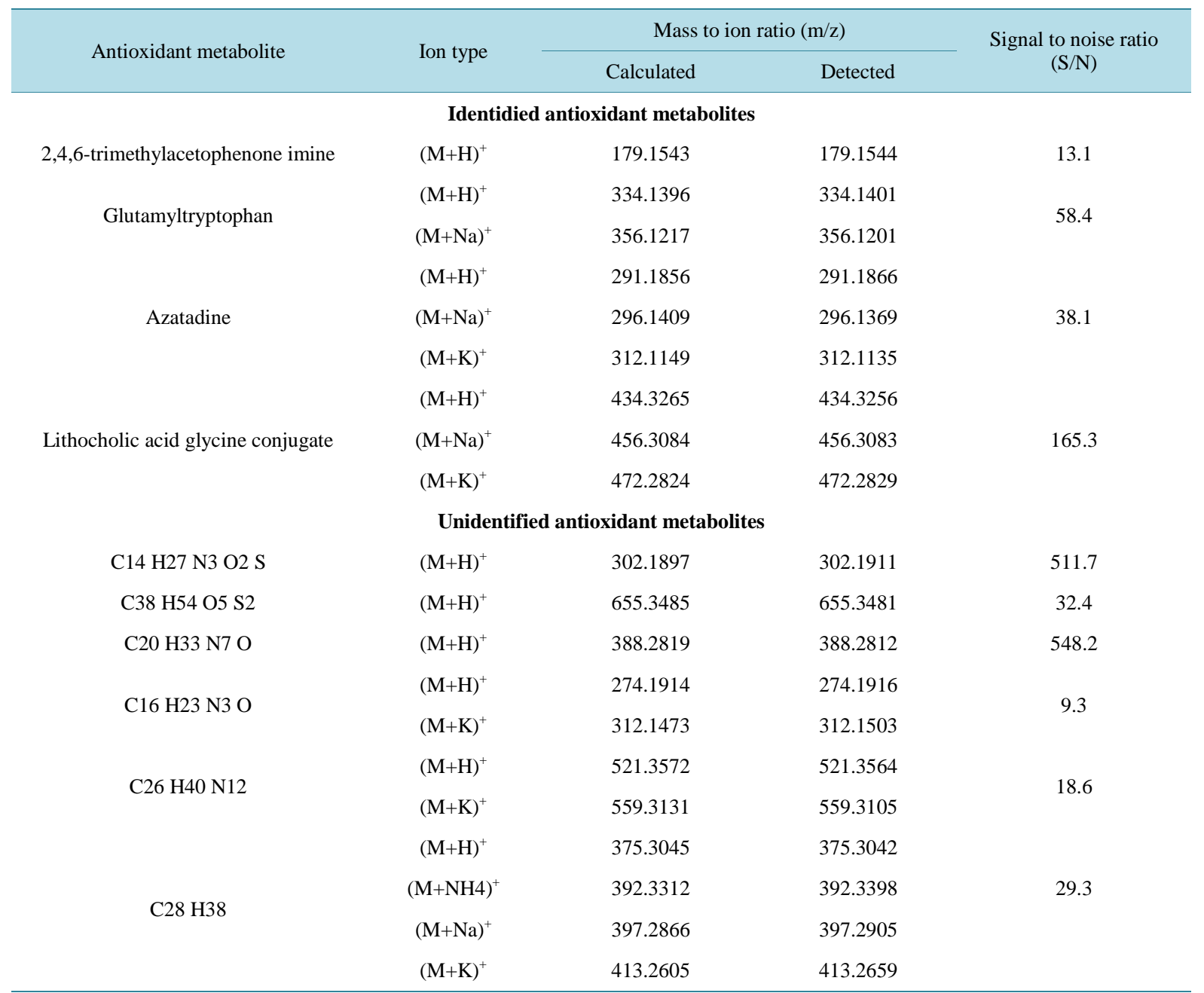

shrooms might give certain level of health defense against uncontrolled production of free radicals. With the established antioxidant activity of this mushroom extracts, an individual activity test of these antioxidative components from Boletus spp. is highly recommended.

\section{Acknowledgements}

This study was financially supported by Ministry of High Education, Malaysia (Grant \# FRGS/1/2013/ST03/ UKM/02/4). The authors would like to thank Dr. Mohd Nazir Basiran Agro-Biotechnology Institute, Malaysia (ABI) executive director for facilitating LC-QTOF-MS work. Special thanks to Pharmacogenomics Center (PROMISE) at Universiti Teknologi MARA (UiTM) for their help and assist.

\section{References}

[1] Wu, X.J. and Hansen, C. (2008) Antioxidant Capacity, Phenolic Content, and Polysaccharide Content of Lentinusedodes Grown in Whey Permeate-Based Submerged Culture. Journal of Food Science, 73, M1-M8. http://dx.doi.org/10.1111/j.1750-3841.2007.00595.x

[2] Coyle, J.T. and Puttfarcken, P. (1993) Oxidative Stress, Glutamate and Neurodegenerative Disorders. Science, 262, 689-695. http://dx.doi.org/10.1126/science.7901908

[3] Kaul, N. and Forman, H.J. (1996) Activation of NFкB by the Respiratory Burst of Macrophages. Free Radical Biology and Medicine, 21, 401-405. http://dx.doi.org/10.1016/0891-5849(96)00178-5

[4] Margaill, I., Plotkine, K. and Lerouet, D. (2005) Antioxidant Strategies in the Treatment of Stroke. Free Radical Biol- 
ogy and Medicine, 39, 429-443. http://dx.doi.org/10.1016/j.freeradbiomed.2005.05.003

[5] Roberts, J.M. and Hubel, C.A. (1999) Is Oxidative Stress the Link in the Two-Stage Model of Preeclampsia? Lancet, 354, 788-789. http://dx.doi.org/10.1016/S0140-6736(99)80002-6

[6] Hamid, A.A., Aiyelaagbe, O.O., Usman, L.A., Ameen, O.M. and Lawal, A. (2010) Antioxidants: Its Medicinal and Pharmacological Applications. African Journal of Pure and Applied Chemistry, 4, 142-151.

[7] Djeridane, A., Yousfi, M., Nadjemi, B., Boutassouna, D., Stocker, P. and Vidal, N. (2006) Antioxidant Activity of Some Algerian Medicinal Plants Extracts Containing Phenolic Compounds. Food Chemistry, 97, 654-660. http://dx.doi.org/10.1016/j. foodchem.2005.04.028

[8] Wong, J., Ng, T.B., Cheung, R.F., Ye, X.J., Wang, H.X. and Lam, S.K., et al. (2010) Proteins with Antifungal Properties and Other Medicinal Applications from Plants and Mushrooms. Applied Microbiology and Biotechnology, 87, 1221-1235. http://dx.doi.org/doi:10.1007/s00253-010-2690-4

[9] Tidke, G. and Rai, M.K. (2006) Biotechnological Potential of Mushrooms: Drugs and Dye Production. International Journal of Medicinal Mushrooms, 8, 351-360. http://dx.doi.org/doi:10.1615/IntJMedMushr.v8.i4.60

[10] Kawagishi, H. (2003) Functional Mushrooms and Their Active Principles. Food Style, 7, 70-73.

[11] Kawagishi, H., Ando, M., Mizuno, T., Yokota, H. and Konishi, S. (1990) A Novel Fatty Acid from the Mushroom Hericium erinaceum. Agricultural and Biological Chemistry, 54, 1329-1331. http://dx.doi.org/10.1271/bbb1961.54.1329

[12] Bernas, E., Jaworska, G. and Lisiewska, Z. (2006) Edible Mushrooms as a Source of Valuable Nutritive Constituents. Acta Scientiarum Polonorum, Technologia Alimentaria, 5, 5-20.

[13] Kirk, P.M., Cannon, P.F., Minter, D.W. and Stalpers, J.A. (2008) Dictionary of the Fungi. 10th Edition, CAB International, Wallingford.

[14] Kuka, M. and Cakste, I. (2011) Bioactive Compounds in Latvian Wild Edible Mushroom Boletus edulis. Proceedings of the 6th Baltic Conference on Food Science and Technology, Jelgava, 5-6 May 2011, 116-120.

[15] Kasuga, A., Aoyagi, Y. and Sugahara, T. (1995) Antioxidant Activity of Fungus Suihs bovinus (L: Fr.) O. Kuntze. Journal of Food Science, 60, 1113-1115. http://dx.doi.org/10.1111/j.1365-2621.1995.tb06304.x

[16] Singleton, V.L. and Rossi, J.A. (1965) Colorimetry of Total Phenolics with Phosphomolybdic-Phosphotungstic Acid Reagents. American Journal of Enology and Viticulture, 16, 144-158.

[17] Wong, J.Y. and Chye, F.Y. (2009) Antioxidant Properties of Selected Tropical Wild Edible Mushrooms. Journal of Food Composition and Analysis, 22, 269-277. http://dx.doi.org/10.1016/j.jfca.2008.11.021

[18] Calderón, A.I., Wright, B.J., Hurst, W.J. and van Breemen, R.B. (2009) Screening Antioxidants Using LC-MS: Case Study with Cocoa. Journal of Agricultural and Food Chemistry, 57, 5693-5699. http://dx.doi.org/10.1021/jf9014203

[19] Mujic, I., Zekovic, Z., Lepojevic, Z., Vidovic, S. and Zikovic, J. (2010) Antioxidant Properties of Selected Edible Mushroom Species. Journal of Central European Agriculture, 11, 389-392.

[20] Gummer, J.A., Krill, C., Du Fall, L., Waters, O.C., Trengove, R., Oliver, R. and Solomon, P. (2012) Metabolomics Protocols for Filamentous Fungi. In: Bolton, M.D. and Thomma, B.P.H.J., Eds., Plant Fungal Pathogens: Methods and Protocols, Humana Press, New York, 237-254. http://dx.doi.org/10.1007/978-1-61779-501-5_15

[21] Hajji, M., Jarraya, R., Lassoued, I., Masmoudi, O., Damak, M. and Nasri, M. (2010) GC/MS and LC/MS Analysis, and Antioxidant and Antimicrobial Activities of Various Solvent Extracts from Mirabilis jalapa Tubers. Process Biochemistry, 45, 1486-1493. http://dx.doi.org/10.1016/j.procbio.2010.05.027

[22] Vaclavik, L., Lacina, O., Hajslova, J. and Zweigenbaum, J. (2011) The Use of High Performance Liquid Chromatography-Quadrupole Time-of-Flight Mass Spectrometry Coupled to Advanced Data Mining and Chemometric Tools for Discrimination and Classification of Red Wines According to Their Variety. Analytica Chimica Acta, 685, 45-51. http://dx.doi.org/10.1016/j.aca.2010.11.018

[23] Cheung, L.M., Cheung, P.C.K. and Ooi, V.E.C. (2003) Antioxidant Activity and Total Phenolics of Edible Mushroom Extracts. Food Chemistry, 81, 249-255. http://dx.doi.org/10.1016/S0308-8146(02)00419-3

[24] Molyneux, P. (2004) The Use of Stable Free Radical Diphenylpicrylhydrazyl (DPPH) for Estimating Antioxidant Activity. Songklanakarin Journal of Science and Technology, 26, 211-219.

[25] Heleno, S.A., Barros, L., Sousa, M.J., Martins, A., Santos-Buelga, C. and Ferreira, I.C.F.R. (2011) Targeted Metabolites Analysis in Wild Boletus Species. LWT-Food Science and Technology, 44, 1343-1348. http://dx.doi.org/10.1016/j.lwt.2011.01.017

[26] Eriksson, L., Johansson, E., Kettaneh-Wold, N., Trygg, J., Wikstrom, C. and Wold, S. (2006) Multi- and Megavariate Data Analysis, Part 1: Basic Principles and Applications. 2nd Edition, Umetrics AB, Umea, 63-98. 